\title{
The effect of $\gamma^{\prime}$ particle size on the deformation mechanism in an advanced polycrystalline nickel-base superalloy
}

\author{
Michael Preuss ${ }^{1}$, Joao Quinta da Fonseca ${ }^{1}$, Benedict Grant ${ }^{1}$, Elisabeth Knoche ${ }^{1}$, Richard Moat ${ }^{1}$ and Mark Daymond ${ }^{2}$ \\ ${ }^{1}$ The University of Manchester, School of Materials, Grosvenor St, Manchester, M1 7HS, UK \\ ${ }^{2}$ Dept. of Mechanical and Materials Engineering, Queen's University, Kingston, K7L 3N6, Canada
}

Keywords: Deformation mechanisms, $\gamma^{\prime}$ particle size, load partitioning, neutron diffraction, electron microscopy

\begin{abstract}
The deformation mechanisms under tensile loading in a $48 \mathrm{vol} . \%$ $\gamma^{\prime}$ polycrystalline nickel-base superalloy (RR1000) have been studied in-situ using neutron diffraction at $20^{\circ} \mathrm{C}, 500^{\circ} \mathrm{C}$ and $750^{\circ} \mathrm{C}$. In addition, post-mortem microstructural studies were carried out on deformed samples using an ultra high resolution field emission gun scanning electron microscope (FEGSEM). Deformation studies were carried out on three different model microstructures with a uni-modal $\gamma^{\prime}$ mean particle size of $80 \mathrm{~nm}$, $120 \mathrm{~nm}$ and $250 \mathrm{~nm}$. The elastic response of $\gamma$ and $\gamma^{\prime}$ during in-situ loading was measured by neutron diffraction and load transfer from $\gamma$ to $\gamma^{\prime}$ was observed during plastic deformation at high temperature in samples with a coarse $\gamma^{\prime}$ mean particle size. It was found that as the testing temperature increases, load transfer can be observed first only for the coarse $\gamma^{\prime}$ microstructure and at $750^{\circ} \mathrm{C}$ for the medium and coarse $\gamma^{\prime}$ microstructure showing that there is a combined particle size/temperature dependency for $\gamma$ to $\gamma^{\prime}$ load transfer. No significant load transfer was detectable in samples with a fine mean $\gamma^{\prime}$ particle size at any temperature. In some cases a region of plastic deformation without load transfer was succeeded by $\gamma$ to $\gamma^{\prime}$ load transfer when a certain level of plastic straining had been exceeded. FEGSEM studies of the samples plastically deformed at $500{ }^{\circ} \mathrm{C}$ showed sheared particles only in the fine $\gamma^{\prime}$ microstructure but not in samples with coarse $\gamma^{\prime}$. The data recorded during the in-situ loading experiment demonstrate that such experiments are suitable for detecting changes of the deformation mode. But it is only in combination with post mortem electron microscopy studies that the load transfer observed can be related to a specific change of slip mode. So far, the experimental data suggest that fine $\gamma^{\prime}$ is sheared during plastic deformation at room and high temperature up to $750^{\circ} \mathrm{C}$ whereas in coarse $\gamma^{\prime}$ Orowan looping is the most likely deformation mechanism at high temperature although cutting by strongly coupled dislocation might also explain the observed load transfer.
\end{abstract}

\section{Introduction}

In the last 1 to 2 decades, new polycrystalline nickel-base superalloys have been developed for disk applications operating at higher temperatures, containing a significantly higher volume fraction of $\gamma^{\prime}$ than previous superalloys (Waspaloy and IN718). These new alloys (RR1000, Rene104, LSHR etc.) have a $\gamma^{\prime}$ volume fraction of close to $50 \%$ and it is reasonable to assume that classical precipitation strengthening mechanisms fail to provide a full mechanistic understanding since constraint between the two phases during plasticity are not accounted for. Understanding the deformation mechanisms in these alloys is critical, as they must combine good tensile strength and fatigue resistance with improved creep resistance.
To date, the optimisation of the mechanical properties of these alloys through thermomechanical processing has been predominantly empirical. Key to harnessing the full potential of these new high temperature alloys is an improved fundamental understanding of the interplay of $\gamma^{\prime}$-distribution and deformation mechanisms. In nickel-base superalloys, $\gamma^{\prime}$ precipitates are usually coherent with the $\gamma$ matrix and display an exact cube-cube orientation relationship with the $\gamma$ grains [1]. The unstressed lattice parameter of the $\gamma^{\prime}$ precipitates is generally slightly different to that of the $\gamma$ matrix although the lattice misfit is often not well determined in polycrystalline nickel-base superalloys due to experimental difficulties. Recent work using neutron and high energy synchrotron $\mathrm{x}$-ray diffraction has demonstrated that when comparing some high $\gamma^{\prime}$ volume fraction nickel-base superalloys, significant differences in lattice misfit and coherency strain can be observed and that the degree of misfit is dependent on cooling rates and aging procedures $[2,3,4]$.

The precipitation hardening mechanisms of $\gamma^{\prime}$-strengthened nickel-base superalloys have been studied in great detail by a number of researchers $[5,6,7]$. It is now generally accepted that with increasing $\gamma^{\prime}$ precipitate size, the deformation mechanism changes from cutting by weakly paired dislocations to strongly paired dislocations, and that large $\gamma^{\prime}$ precipitates are bypassed by Orowan looping of dislocations. However, very little understanding exists of how these trends are affected by the testing temperature. It is well known that the $\mathrm{L}_{2}$ ordered $\gamma^{\prime}$ structure displays an anomalous yield stress versus temperature behaviour, with Westbrook [8] first showing that $\mathrm{Ni}_{3} \mathrm{Al}$ displays a peak hardness at elevated temperature. The most widely accepted mechanism for the flow stress increase was proposed by Thornton et al. [9] and involves temperature assisted cross slip of screw dislocations from (111) slip planes to the (010) planes where the dislocations are assumed to be immobile. This interaction can result in the unusual behaviour of the high temperature (e.g. $550^{\circ} \mathrm{C}$ ) yield stress being higher than the room temperature value even though the strength of the $\gamma$ matrix decreases with increasing temperature. Therefore, below the peak strength temperature, i.e. in the regime where the yield strength increases with rising temperature, the active slip systems are predominantly $\{111\}<110>$, while above the peak temperature cross slip of $\{100\}<110>$ occurs in $\gamma^{\prime}$ where the dislocations become immobile [10]. The driving force for the change of slip mode with increasing temperature is provided both by the anisotropy of the antiphase boundary (APB) and by the relative change of critical resolved shear stress of the $\{100\}<110>$ system. It was demonstrated by a number of researchers $[11,12]$ that at high temperature, dislocations have a lower energy on the (010) plane than the (111) plane because the APB energy is at a minimum on the (010) plane. Most of the experimental evidence of the change of the slip mode in $\gamma^{\prime}$ has been achieved by in-situ transmission electron microscopy (TEM) studies on single crystal nickel-base 
superalloys [13]. More recently, results obtained from in-situ neutron diffraction studies carried out during tensile loading of Udimet 720Li have shown clear changes in the elastic response of the $\gamma$ and $\gamma^{\prime}$ phase during loading beyond the yield point as the testing temperature was increased beyond $500^{\circ} \mathrm{C}$ [14]. The interpretation of the experimental data was supported by a new two-site elastoplastic self-consistent (EPSC) polycrystal deformation model. The results demonstrated that the change of slip mode in $\gamma^{\prime}$ with increasing temperature in polycrystalline nickel-base superalloys can be detected by the combination of insitu neutron diffraction studies and EPSC modelling.

In many advanced nickel-base superalloys, the complexity of the $\gamma^{\prime}$ distribution makes studying the fundamental deformation mechanisms very difficult. Typical high $\gamma^{\prime}$ volume fraction nickel-base superalloys display a bi-modal or tri-modal $\gamma^{\prime}$ size distribution because of the requirement to cool large forgings with a relatively slow cooling rate when being heat-treated either above or slightly below the $\gamma^{\prime}$-solvus. Fast cooling rates would result in unacceptable large residual stresses and would also increase the susceptibility of quench cracking [15]. However, characterisation of RR1000 weld microstructures has demonstrated that uni-modal $\gamma^{\prime}$ distribution can be achieved in alloys such as RR1000 when sufficiently high cooling rates are applied [16].

In the present work, neutron diffraction in-situ deformation studies were carried out on three uni-modal $\gamma^{\prime}$ distributions in RR1000 between room temperature and $750^{\circ} \mathrm{C}$. These measurements provided data on the elastic response of the $\gamma$ and the $\gamma^{\prime}$ phase (presented here) and the diffraction elastic strain of grain families (not included here). The results demonstrate that this experimental approach can help understand the deformation mechanisms in $\gamma^{\prime}$ strengthened nickel-base superalloys by observing in-situ the load partitioning between the two phases as a function of test temperature, amount of plastic deformation and $\gamma^{\prime}$ particle size. These findings are supplemented by FEGSEM studies on the deformed samples.

\section{Material}

The material studied is RR1000, a nickel-base superalloy produced by a powder metallurgical route followed by forging. The chemical composition of RR1000 can be found in Table 1 . The material was provided by Rolls-Royce plc. in a sub-solvus heat treated fully aged condition with a complex tri-modal $\gamma^{\prime}$ distribution and an approximately random texture. In order to identify the effect of $\gamma^{\prime}$ particle size on the dominance of particular deformation mechanisms, three different uni-modal $\gamma^{\prime}$ distributions were generated. Small blanks of about $12 \times 12 \times$ $80 \mathrm{~mm}^{3}$ were heat treated in a tube furnace under argon atmosphere for 2 hours at about $20^{\circ} \mathrm{C}$ above the $\gamma^{\prime}$-solvus followed by oil quenching. FEGSE microscopy showed that, following quenching, the material contained fine $\gamma^{\prime}$ particles of about $50 \mathrm{~nm}$ in size. Different mean $\gamma^{\prime}$ particle sizes were then generated by heat treating the quenched material for one hour at either $800^{\circ} \mathrm{C}, 900$ or $1050^{\circ} \mathrm{C}$, followed by slow cooling $(0.1-$ $1{ }^{\circ} \mathrm{C} / \mathrm{min}$ ) in order to minimise the possibility of secondary $\gamma^{\prime}$ precipitation. These slow cooling rates were also aimed at minimising chemical differences between fine, medium and coarse $\gamma$ in order to avoid significant variations of $\gamma / \gamma^{\prime}$ lattice misfit for the three microstructures.
Table1: Nominal chemical composition (wt $\%$ ) of RR1000:

\begin{tabular}{|c|c|c|c|c|c|c|c|c|c|}
\hline $\mathrm{Ni}$ & $\mathrm{Cr}$ & $\mathrm{Co}$ & $\mathrm{Mo}$ & $\mathrm{Al}$ & $\mathrm{Ti}$ & $\mathrm{Ta}$ & $\mathrm{Zr}$ & $\mathrm{C}$ & $\mathrm{B}$ \\
\hline bal & 15 & 18.5 & 5 & 3 & 3.6 & 2 & 0.6 & 0.027 & 0.015 \\
\hline
\end{tabular}

\section{Experimental Methods}

Neutron diffraction can be used to determine the average phase elastic strain or the elastic strains in particular orientations of grains in a polycrystalline metal. In these neutron diffraction experiments, a typical volume of several $\mathrm{mm}^{3}$ is defined using collimators on the incident and diffracted beam paths; thus providing measurements of elastic strain averaged over several thousand grains. The method is well established and is described in, for example $[17,18]$. Here we emphasize that such a diffraction measurement involves the monitoring of changes in separation of one or more suitably orientated crystallographic lattice planes. It is thus a direct measure of the elastic strain in the material. In a polycrystal, the lattice spacing, obtained by fitting a diffraction peak, represents the average lattice separation over all the grains in the irradiated volume that are suitably oriented to diffract.

A series of increasing uniaxial tensile loads were applied sequentially to each specimen in-situ on the ENGIN-X instrument [19] at the ISIS pulsed neutron facility, Rutherford Appleton Laboratory, UK. The load frame and its use is described in detail elsewhere [20], but in brief the loading axis is horizontal and at $45^{\circ}$ to the incident beam, allowing simultaneous measurement of lattice plane spacings both parallel and perpendicular to the loading direction. The optical furnace used to heat the samples, in air, is described in more detail in [21]. The test temperature during each loading experiment was measured using a $\mathrm{K}$ type thermocouple held in contact with the sample, just outside the diffracting gauge volume. In-situ loading experiments were carried out at temperatures of 20,500 and $750^{\circ} \mathrm{C}$.

At time-of-flight sources, such as ISIS, neutron pulses, each with a continuous range of velocities and therefore wavelengths, are directed at a specimen. The flight times of diffracted neutrons are measured, allowing calculation of wavelengths, and the recording of diffraction spectra. The incident spectra are polychromatic, thus many lattice planes are recorded in each measurement. The scattering vectors for all reflections recorded in one detector lie in the same direction, and thus indicate the strain in that direction in the sample. Each reflection is produced from a different family of grains, oriented such that a specific $h k l$ plane diffracts to the detector. A more complete description of these issues can be found elsewhere [22]. It is now common practice at such a source to fit the whole spectrum simultaneously using a Rietveld refinement [23] when investigating the 'average' or continuum equivalent strain in a phase. A two phase Rietveld refinement was used to produce the phase average strains in each direction, parallel (longitudinal) and perpendicular (transverse) to the loading axis respectively. The diffraction data analysis in RR1000 is complicated by $\gamma(\mathrm{fcc})$ and $\gamma^{\prime}\left(\mathrm{L}_{2}\right)$ exhibiting almost identical lattice parameters. The analysis of diffraction data was performed using General Structural Analysis System (GSAS) developed at Los Alamos National Laboratory. The peak positions were initially estimated from the crystal structure and an initial lattice parameter. Debye-Waller thermal factors for each of the alloying elements were taken from Sears and Shelley [24]. For each individual tensile test, the diffraction pattern of the first data point (zero load) was manually fitted. In this case, for each peak position a profile was fitted, which is a convolution between two 
back-to-back exponentials and a pseudo-Voigt function $[25,26]$. The intensity for each of the peaks was determined from the crystal structure and instrument parameters. The volume fraction of $\gamma$ and $\gamma^{\prime}$ was kept constrained and the lattice parameters, profile shape coefficients and elastic anisotropy were allowed to converge using a least-squares fit. The subsequent diffraction patterns were fitted using the same fitting process and using the fitting results from the previous measurement point as initial guess. Single peak fits of particular grain families were also carried out but are not presented here. As mentioned above, the two phases display a slight lattice mismatch that is known to contribute to the overall strength in single-crystal nickel-base superalloys [27]. However, in polycrystalline Ni superalloys, the influence of the $\gamma / \gamma^{\prime}$ lattice mismatch on mechanical properties is far less studied than in single crystal material. As we measured the lattice spacing of each phase during the in-situ loading experiments, we report the evolution of the $\gamma / \gamma^{\prime}$ lattice mismatch during loading.

Tensile test were not carried out under continuous loading, as high quality diffraction patterns on ENGIN-X require an acquisition time of about 20 minutes for RR1000. Instead, the sample was loaded stepwise and constant stress levels were maintained within the elastic and plastic regime by setting the stress rig to stress control. Macroscopic strain was monitored on the samples using a dynamic high temperature extensometer clip gauge in all cases. A slit $6 \mathrm{~mm}$ high, $4.5 \mathrm{~mm}$ wide defined the incident beam, while radial collimators provided a scattered beam width of $4.5 \mathrm{~mm}$. In each case the strains shown are with respect to the initial lattice parameter measured at a nominal zero stress, for the given temperature. That is, strain $\varepsilon=\left(a-a_{0}\right) / a_{0}$ where the lattice parameter $a$ of each phase is determined by Rietveld refinement and $a_{0}$ is the starting value. Therefore, the strains reported do not show any possible contribution to the initial elastic strain from thermomechanical processing.

With the significant improvement in scanning electron microscopy it is now possible to characterize even very fine $\gamma^{\prime}$ precipitates by etching polished samples according to [16] and using a state-of-the-art FEGSEM. For the present work a FEI Sirion FEGSEM was used to study the $\gamma^{\prime}$ precipitation size of the three different microstructural conditions and to identify any sheared $\gamma^{\prime}$ in the deformed samples. This post mortem characterization was carried out on the tested tensile samples, sectioned along the loading direction and subsequently etched in the same way.

In order to determine the mean precipitation size of the uni-modal $\gamma^{\prime}$ distributions, image analysis was carried out using the Kontron KS 400 software package.

\section{Results and Discussion}

Microstructure:

Figure 1 displays the typical $\gamma^{\prime}$ distributions of the fine, medium and coarse $\gamma^{\prime}$ microstructures. The aim was to achieve a uni-modal $\gamma^{\prime}$ distribution to subsequently study the effect of $\gamma^{\prime}$ particle size on deformation mechanism and not to develop optimized microstructures for service conditions. It should be noted that a uni-modal $\gamma^{\prime}$ distribution with an average particle size of around $50 \mathrm{~nm}$ was already observed after oil quenching. Consequently, a coarse $\gamma^{\prime}$ distribution was achieved by aging the material at a very high temperature, which partly dissolved $\gamma^{\prime}$ and resulted in
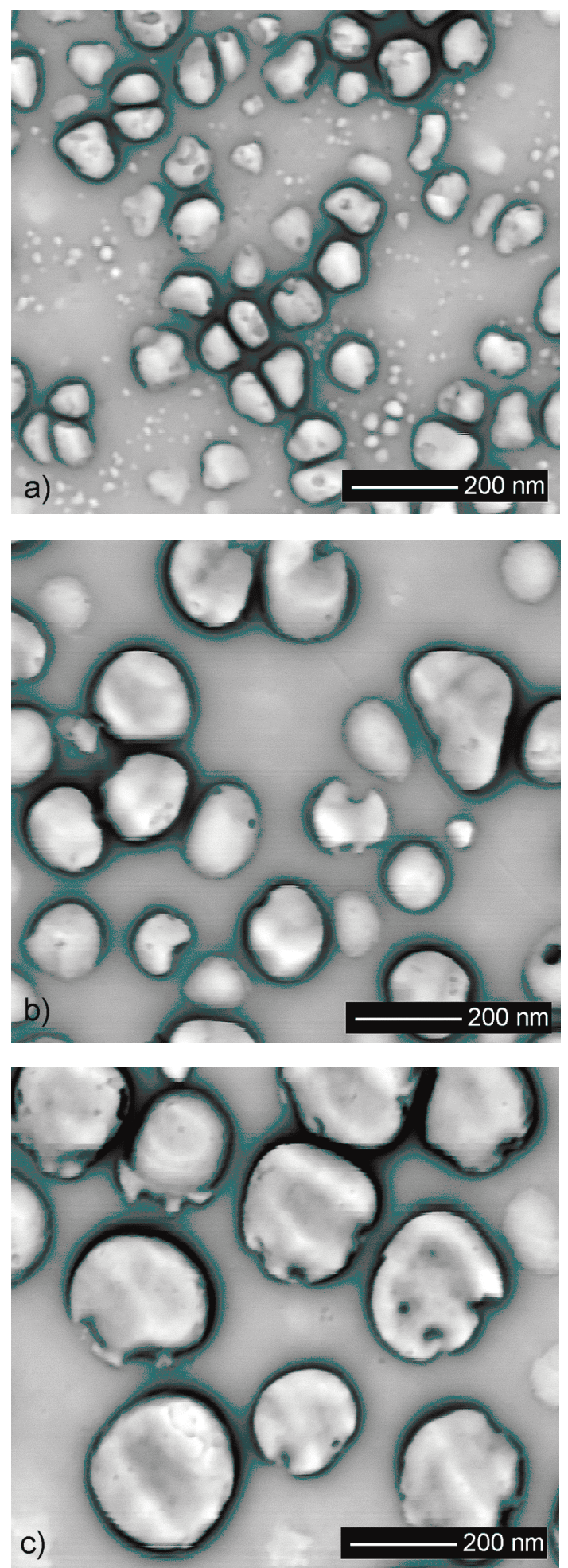

Figure 1: $\gamma^{\prime}$ distributions of the fine (a), medium (b) and coarse (c) $\gamma^{\prime}$ microstructure. Note that apart from (a) fairly uni-modal $\gamma^{\prime}$ distributions were achieved. 
coarsening of the remaining $\gamma^{\prime}$ during high temperature aging and during very slow cooling to room temperature $\left(1^{\circ} \mathrm{C} / \mathrm{min}\right.$ down to $900^{\circ} \mathrm{C}$ followed by $0.1^{\circ} \mathrm{C} / \mathrm{min}$ down to $500^{\circ} \mathrm{C}$ followed by air cooling). It can be seen from Figure 1 that uni-modal $\gamma^{\prime}$ distributions were achieved in the case of the material heat treated at $900^{\circ} \mathrm{C}$ (Figure $1 \mathrm{~b}$ ) and $1050{ }^{\circ} \mathrm{C}$ (Figure 1c) but not for the fine $\gamma^{\prime}$ microstructure (Figure 1a). In this case very fine $\gamma^{\prime}$ had formed around the primary $\gamma^{\prime}$. The origin of the bi-modal $\gamma^{\prime}$ distribution after aging at $800^{\circ} \mathrm{C}$ is not entirely clear but might be related to unwanted precipitation during the relatively fast cooling $\left(1^{\circ} \mathrm{C} / \mathrm{min}\right)$ employed following aging. In contrast, all other samples were cooled with $0.1^{\circ} \mathrm{C} / \mathrm{min}$ to $500^{\circ} \mathrm{C}$ before air-cooled. The average $\gamma^{\prime}$ particle sizes were determined to be $80 \mathrm{~nm}(15 \mathrm{~nm}$ for the finer intervening particles) for the fine, $120 \mathrm{~nm}$ for the medium and $250 \mathrm{~nm}$ for the coarse $\gamma^{\prime}$ microstructure. In all cases the $\gamma^{\prime}$ morphology appeared fairly spherical.

The $\gamma / \gamma^{\prime}$ lattice misfit was determined for each microstructure at the three testing temperatures during the first measurement in the unloaded condition of the in-situ loading/neutron diffraction experiment. The lattice misfit was calculated using the following equation, where $a$ is the lattice parameter:

$$
\delta=\frac{2 \cdot a\left(\gamma^{\prime}\right)-a(\gamma)}{a\left(\gamma^{\prime}\right)-a(\gamma)}
$$

The lattice misfits measured in the loading and transverse direction at zero load were generally in excellent agreement. Figure 2 plots the averaged lattice misfit measured in the two different directions as a function of temperature for the three different microstructures. In addition, Figure 2 also displays lattice misfit data recorded during an in-situ loading experiment of Udimet 720Li [14]. In this case, the alloy was tested in a standard fully heat treated condition. Firstly, it can be seen that apart from the fine $\gamma^{\prime}$ microstructure tested at $500^{\circ} \mathrm{C}$, there is negligible variation of lattice misfit between the three different microstructures, when compared for a particular testing temperature. It is interesting to note that for the tested temperature range, the model microstructures of RR1000 exhibit a lattice misfit almost 3 times smaller than Udimet $720 \mathrm{Li}$. At a testing

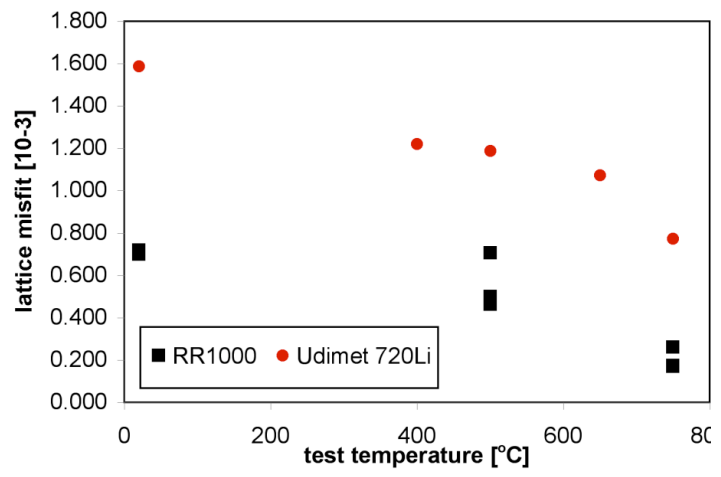

Figure 2: Lattice misfit between $\gamma$ and $\gamma^{\prime}$ as a function of temperature for RR1000 and Udimet 720Li [14]. In the case of Udimet $720 \mathrm{Li}$ only a standard microstructure was tested while in the case of RR1000 each data point for a certain test temperature represents a different uni-modal $\gamma^{\prime}$ microstructure (fine, medium, coarse). temperature of $750^{\circ} \mathrm{C}$, the lattice misfit observed in RR1000 is almost zero.

Stress - macrostrain behavior:

Figure 3a-c shows plots of true stress/true strain curves recorded during the in-situ tensile loading experiment on ENGIN-X. It should be remembered at this stage that these were not continuous loading experiments. Instead, the samples were loaded with a strain rate of about $10^{-5} \mathrm{~s}^{-1}$ to a certain stress level before changing to stress control in order to keep the stress level constant during the diffraction experiment. Macroscopic strain was monitored on
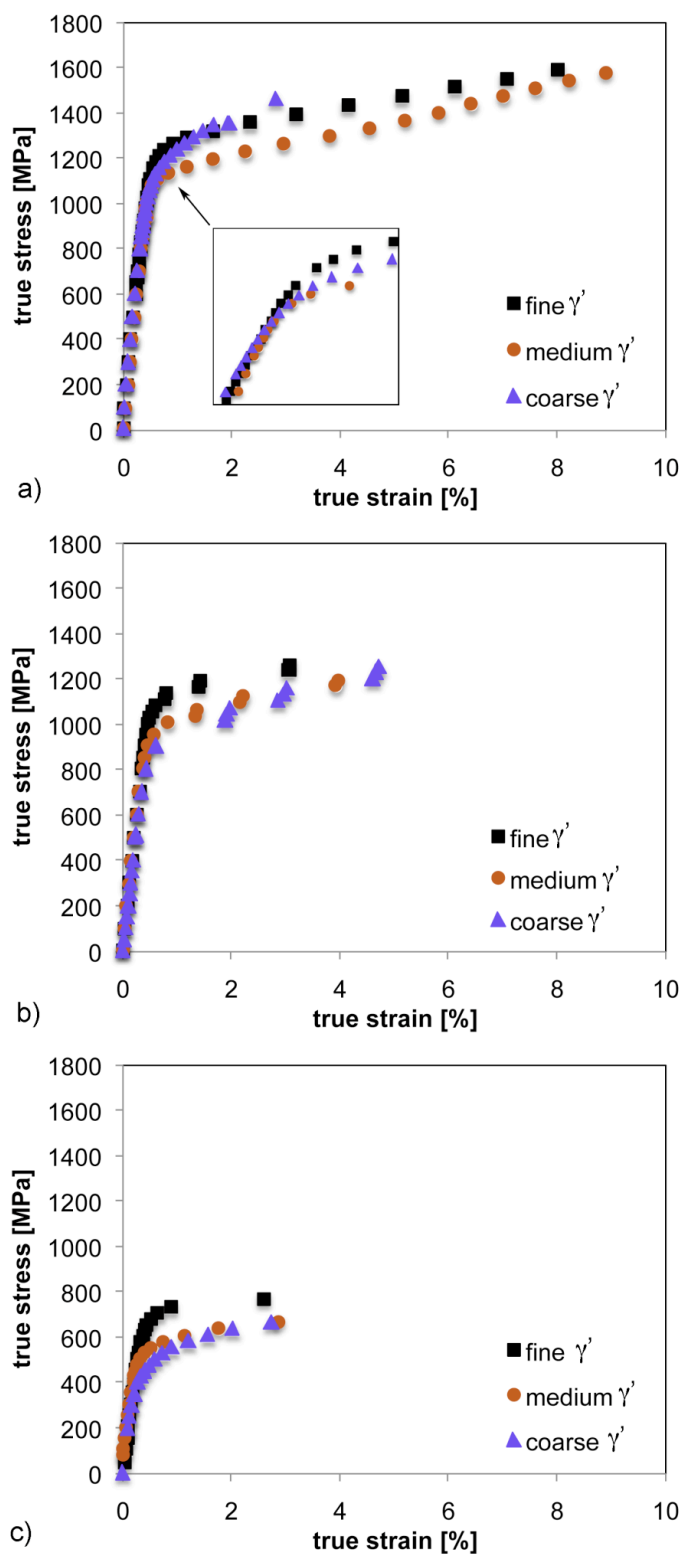

Figure 3: Stress - strain curves of RR1000 with a model microstructure of fine, medium and coarse $\gamma^{\prime}$ tested at (a) room temperature, (b) $500^{\circ} \mathrm{C}$ and (c) $750^{\circ} \mathrm{C}$. The fine $\gamma^{\prime}$ microstructure shows the highest yield point at all testing temperatures. 
the samples throughout the experiment. At stresses above the yield point, plastic deformation was generally observed when keeping the samples in stress control. However, the amount of deformation was small and most of it was observed during the first few minutes. For this reason, the neutron diffraction experiment was always started once plastic deformation (creep) had become hardly noticeable, which usually took about 5 minutes. The strain data plotted in Figure 3 is the averaged strain value (converted to true strain) recorded during the duration of one load step. At all testing temperatures, the fine $\gamma^{\prime}$ microstructure showed the highest yield point of the three different microstructures. With increasing testing temperature, this trend became more pronounced. Figure 3 generally exhibits a trend of decreasing yield strength with increasing $\gamma^{\prime}$ particle size. Only when tested at room temperature, the medium and coarse $\gamma^{\prime}$ microstructure showed a similar yield strength to that of the fine $\gamma^{\prime}$ microstructure. At that temperature, the coarse $\gamma^{\prime}$ microstructure also displayed significantly more work hardening during tensile loading than the other two microstructures.

\section{Elastic strain response of $\gamma$ and $\gamma^{\prime}$}

Figures 4-6 display the load partitioning between $\gamma$ and $\gamma^{\prime}$ recorded by neutron diffraction during straining along the loading direction. It is important to note that the lattice shift of $\gamma$ and $\gamma^{\prime}$ recorded by neutron diffraction describes the elastic response of each individual phase during loading the material in the elastic and plastic regime. As long as no load partitioning takes place, both phases are expected to display a similar elastic strain response. However, since the two phases will not have exactly the same Young modulus some slight differences might be observed between the two phases prior to plastic deformation. Once the material starts yielding, many two-phase materials show a load transfer from the soft phase (deforming mostly plastically) towards the hard phase (deforming mostly elastically). Consequently, during a neutron diffraction experiment, the hard phase will show larger elastic strains than the soft phase once the yield point has been exceeded. In the case of a $\gamma^{\prime}$ strengthened nickel-base superalloy, it is fair to assume that shearing of $\gamma^{\prime}$ by weakly coupled dislocations, i.e. simultaneous plastic deformation of $\gamma$ and $\gamma^{\prime}$ will yield no load transfer between the two phases. On the other hand, Orowan looping of dislocations will result in plastic deformation in the $\gamma$ but not in the $\gamma^{\prime}$ phase. Therefore, one might expect to observe load transfer from $\gamma$ towards $\gamma^{\prime}$ when this deformation mechanism is active. It should be pointed out that Orowan looping of dislocations has been rarely reported in polycrystalline nickel-base superalloys because it seems to require a $\gamma^{\prime}$ particle size in excess of 400nm [28]. In terms of cutting $\gamma^{\prime}$ by strongly coupled dislocations, it is not entirely clear whether this mechanism on its own would result in load transfer towards $\gamma^{\prime}$. But since this mechanism requires an increased level of dislocation bowing, because the paired dislocations have to cut the particle together, it might well be that this mechanism also results in some $\gamma$ to $\gamma^{\prime}$ load transfer.

Previous work carried out on Udimet $720 \mathrm{Li}$ with a standard trimodal $\gamma^{\prime}$ size distribution demonstrated that at temperatures below $500^{\circ} \mathrm{C}$ load transfer from $\gamma^{\prime}$ towards $\gamma$ can be observed during the early stage of plastic deformation and that consequently in this temperature regime the CRSS of $\gamma^{\prime}$ is smaller than the CRSS of the $\gamma$ phase [14]. The same work showed that above $500^{\circ} \mathrm{C}$ a reverse load transfer is observed with the load being transferred to the $\gamma^{\prime}$ phase during the early stages of yielding. The variation of the elastic strain response for individual superlattice diffraction peaks of the $\gamma^{\prime}$ phase at different temperatures provided a clear signature, which could be correlated with the change in operating slip mechanism from $\{111\}$ to $\{100\}$ with increasing temperature, which is well documented for single crystal nickel-base superalloys [13].
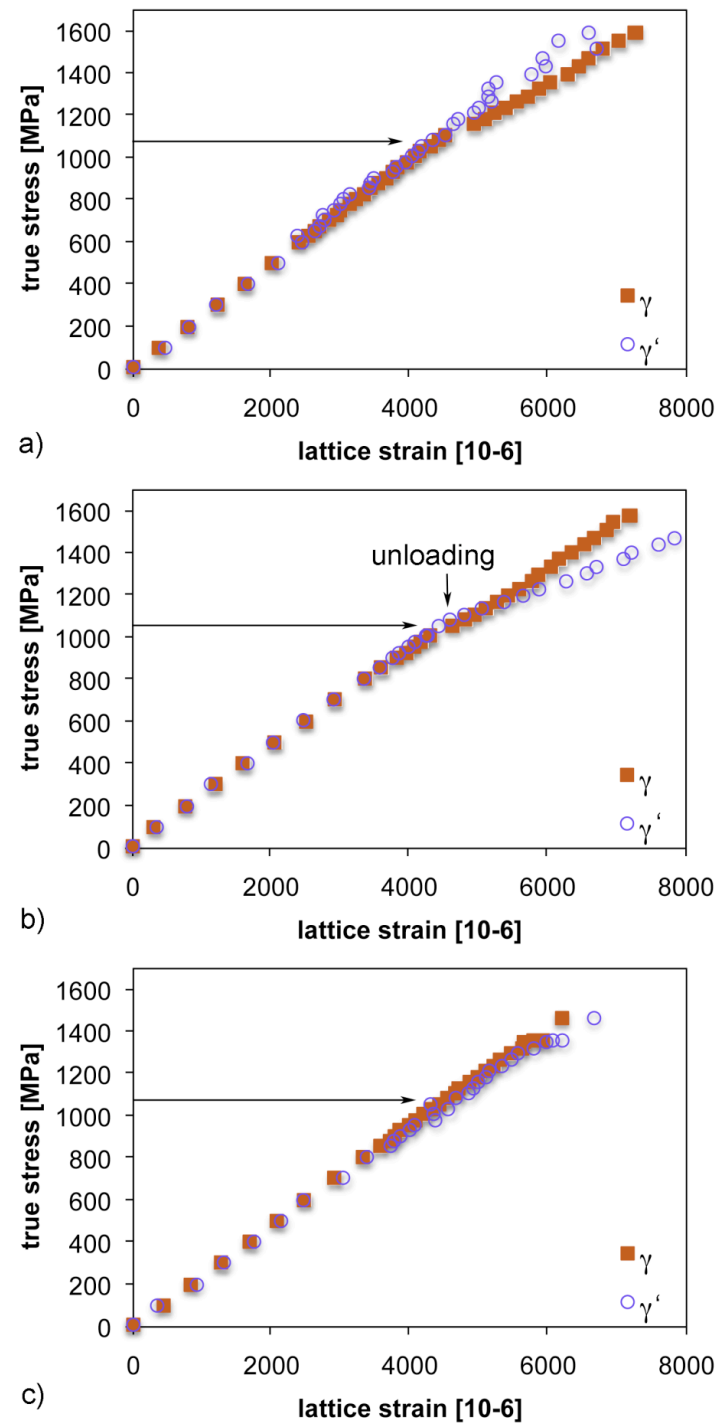

Figure 4: Longitudinal elastic strain response of $\gamma$ and $\gamma^{\prime}$ during loading at room temperature for (a) fine $\gamma^{\prime}$ microstructure, (b) medium $\gamma^{\prime}$ microstructure and (c) coarse $\gamma^{\prime}$ microstructure. The horizontal arrow indicates the yield point of the material estimated from the macroscopic stress - strain curves shown in Figure 3a. In the case of the medium $\gamma^{\prime}$ microstructure the tensile specimen was accidently unloaded at $1100 \mathrm{MPa}$ (about $2 \%$ macrostrain) before continuing the loading experiment. 
Keeping these previous findings in mind, it is now interesting to observe that the fine $\gamma^{\prime}$ microstructure of RR1000 tensile tested at room temperature (Figure 4a) also shows a small load transfer from $\gamma^{\prime}$ to $\gamma$ as previously observed in Udimet 720Li (tri-modal $\gamma^{\prime}$ size distribution), which was also confirmed by the measurements in the transverse direction. The yield point of the tensile specimen estimated from the macroscopic stress - strain curves presented in Figure 3 is indicated with horizontal arrows in Figures 4-6.

In the case of the medium $\gamma^{\prime}$ microstructure tested at room temperature no load transfer was observed up to $1100 \mathrm{MPa}$ (corresponds to almost 3\% macroscopic strain). Upon further

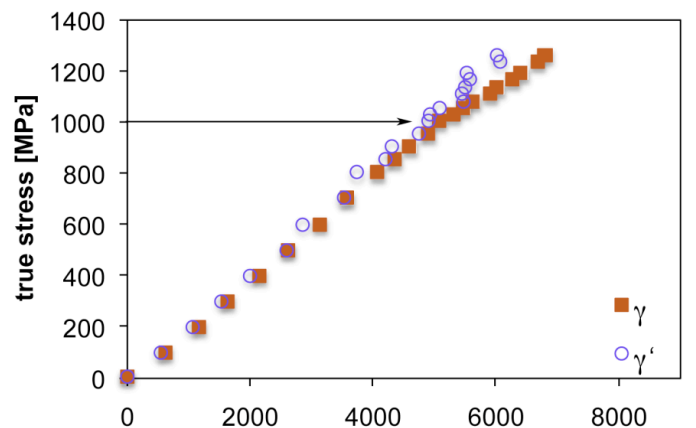

a)

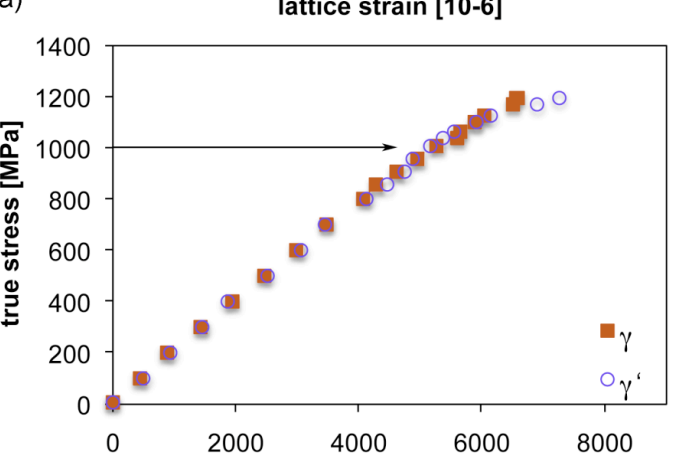

b)

lattice strain [10-6]

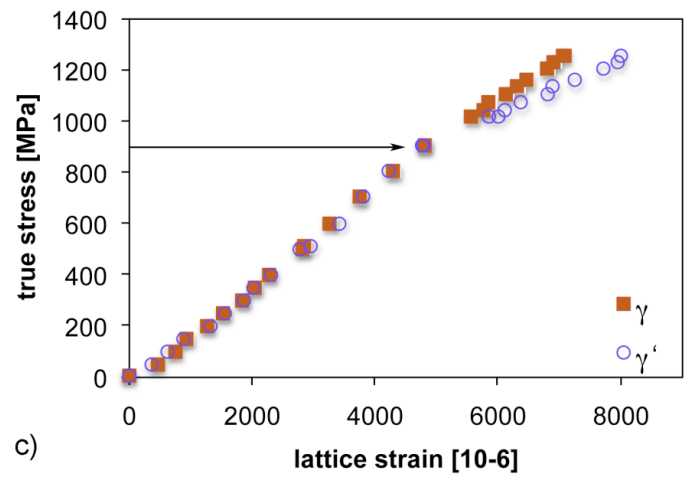

Figure 5: Longitudinal elastic strain response of $\gamma$ and $\gamma^{\prime}$ during loading at room temperature for (a) fine $\gamma^{\prime}$ microstructure, (b) medium $\gamma^{\prime}$ microstructure and (c) coarse $\gamma^{\prime}$ microstructure. The horizontal arrow indicates the yield point of the material estimated from the macroscopic stress - strain curves shown in Figure 3b. loading/straining, load transfer from the $\gamma$ phase to the $\gamma^{\prime}$ phase can be seen (Figure 4b). The same observations were obtained from the measurements in the transverse direction. It can be seen from Figure 3 that only the fine and medium $\gamma^{\prime}$ microstructures tested at room temperature were strained to about $8 \%$ while all other in-situ loading experiments were stopped after about 3\%. The load transfer observed in Figure $4 \mathrm{~b}$ after 3\% strain might suggest that work hardening, i.e. cross slip in the $\gamma^{\prime}$ phase, activates a different deformation mechanism during further yielding. If one assumes that no load transfer to the $\gamma^{\prime}$ phase can
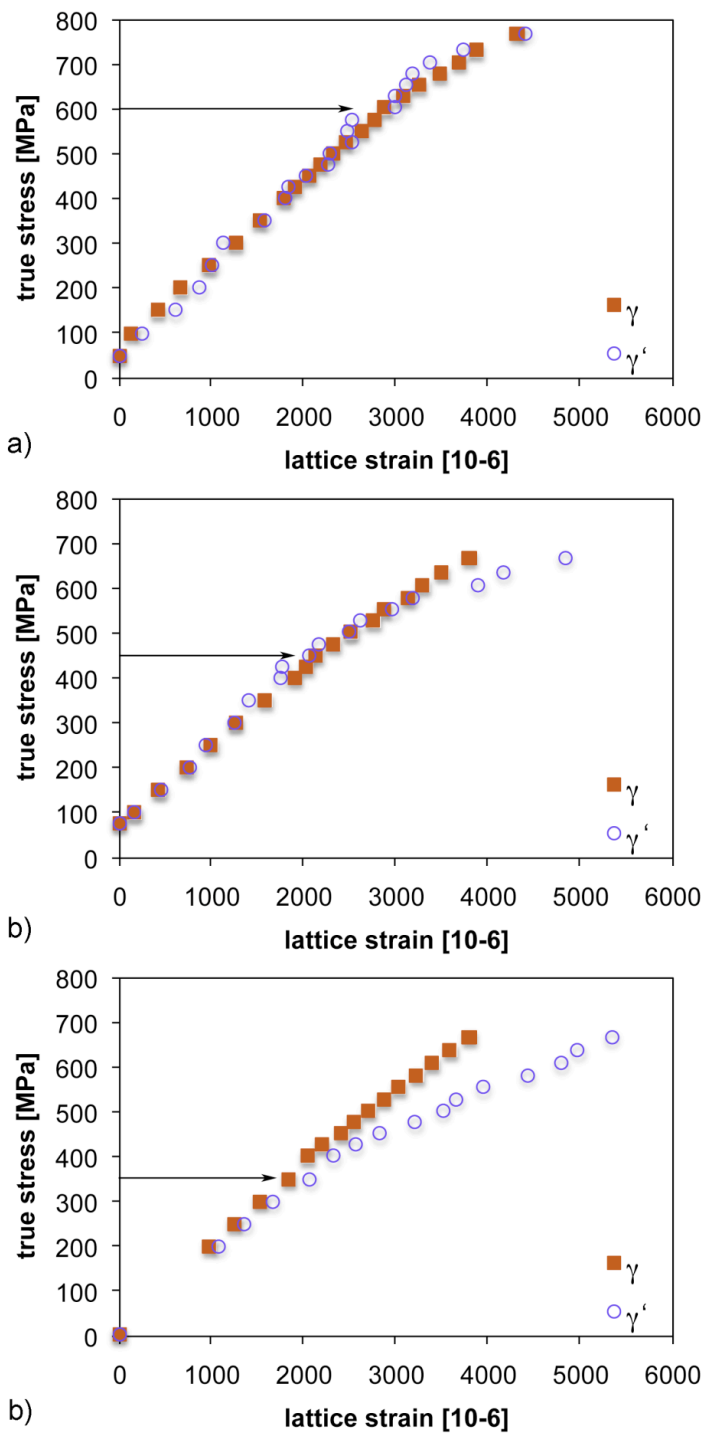

Figure 6: Longitudinal elastic strain response of $\gamma$ and $\gamma^{\prime}$ during loading at room temperature for (a) fine $\gamma^{\prime}$ microstructure, (b) medium $\gamma^{\prime}$ microstructure and (c) coarse $\gamma^{\prime}$ microstructure. The horizontal arrow indicates the yield point of the material estimated from the macroscopic stress - strain curves shown in Figure $3 \mathrm{c}$.

be associated with the cutting of weakly coupled dislocations, 
continued deformation might then result in a change of slip mode to cutting by strongly coupled dislocations or Orowan looping. In the view that work hardening of $\gamma^{\prime}$ should affect cutting of strongly coupled dislocations in the same way it does for weakly coupled dislocations, Orowan looping seems the more plausible mechanism. It should be pointed out that detailed dislocation studies using transmission electron microscopy are impractical when a material has been strained to $8 \%$ plastic deformation.

In the case of the coarse $\gamma^{\prime}$ microstructure tested at room temperature, no load transfer between the two phases was detectable (Figure 4c) when tested to about $2.5 \%$ plastic deformation. The last measurement point was recorded after about $3 \%$ strain and suggests that some load transfer from the $\gamma$ to the $\gamma^{\prime}$ phase starts occurring. Again, these observations were confirmed by the strain response recorded in the transverse direction.

When increasing the test temperature to $500^{\circ} \mathrm{C}$, the fine $\gamma^{\prime}$ microstructure showed a very slight load transfer from the $\gamma$ to the $\gamma^{\prime}$ phase in the longitudinal direction (Figure 5a) and a slight opposite load transfer in the transverse direction. In this case one might assume that overall no load transfer between the two phases takes place and that the observed strain partitioning lies within the range of the experimental accuracy or is a result of the small difference in elastic properties of the two phases. For the medium $\gamma^{\prime}$ microstructure tested at $500^{\circ} \mathrm{C}$ no load transfer was observed in the longitudinal (Figure 5b) and transverse direction until the specimen was macroscopically strained to about $1.5 \%$. After this point slight load partitioning from the $\gamma$ phase to the $\gamma^{\prime}$ phase was observed in both measurement directions suggesting that the $\gamma^{\prime}$ phase exhibits different hardening behavior compared to the matrix. This might be explained by the well-known mechanism of dislocation being locked in the $\gamma^{\prime}$ phase due to cross slip on the $\{100\}$ slip plane [8]. The continued load transfer during further tensile loading might have activated either cutting by strongly coupled dislocations or Orowan looping. Again, Orowan looping seems the more plausible slip mode at this stage. The coarse $\gamma^{\prime}$ microstructure tested at $500^{\circ} \mathrm{C}$ showed load transfer from the $\gamma$ phase to the $\gamma^{\prime}$ phase in the loading (Figure 5c) and transverse direction starting at the onset of macroscopic yielding, which according to [14] can be associated with a change of dominant slip systems in $\gamma^{\prime}$ from $\{111\}<110>$ to $(100\}<110>$ where the dislocations are assumed to be immobile. Following the same line of reasoning now, this locking of dislocations in $\gamma^{\prime}$ should activate Orowan looping and result in the observed load transfer.

At this stage it is interesting to note that the change of slip from the $\{111\}$ plane to the $\{100\}$ plane might not only be dependent on the test temperature but also the $\gamma^{\prime}$ particle size and degree of deformation since for a given temperature it requires higher stresses and more plastic deformation in the medium $\gamma^{\prime}$ microstructure before load transfer from the matrix to the precipitates takes place compared to the coarse $\gamma^{\prime}$ microstructure. In case of the in-situ loading experiment carried out at $750^{\circ} \mathrm{C}$ the $\gamma^{\prime}$ particle size had a similar impact on load transfer as observed in the experiments carried out at $500^{\circ} \mathrm{C}$. However, load sharing for the medium and coarse $\gamma^{\prime}$ microstructures was significantly more pronounced than when testing at lower temperature. For the fine $\gamma^{\prime}$ microstructure only very little $\gamma$ to $\gamma^{\prime}$ load transfer was observed, mainly in the transverse direction, when strained to about 3\%. (Figure 6a shows longitudinal direction). The medium $\gamma^{\prime}$ microstructure showed a more significant load transfer in both measurement directions from the matrix to the $\gamma^{\prime}$ phase but not immediately at the onset of plastic deformation (Figure $6 \mathrm{~b}$ shows the longitudinal direction). However, by far the largest load transfer from the $\gamma$ to the $\gamma^{\prime}$ phase was observed for the coarse $\gamma^{\prime}$ microstructure tested at $750^{\circ} \mathrm{C}$. In this case the load transfer started at the onset of plastic yielding and lattice strain differences of $150010^{-6}$ in the longitudinal and $100010^{-6}$ in the transverse direction were observed after 3\% strain between $\gamma$ and $\gamma^{\prime}$. Similar to the observations made at $500^{\circ} \mathrm{C}$, the in-situ loading experiments carried out at $750^{\circ} \mathrm{C}$ give clear evidence that load transfer from the $\gamma$ to the $\gamma^{\prime}$ phase does not only depend on the testing temperature but also on the degree of macroscopic deformation and $\gamma^{\prime}$ particle size. In terms of particle size dependency, one would expect Orowan looping to be active when the $\gamma^{\prime}$ particles have reached a critical size. On the other hand Orowan loops are rarely seen in turbine disk material $[6,29]$, which might well be related to the generally low lattice misfit between $\gamma$ and $\gamma^{\prime}$ in such alloys. The complex bi-modal or trimodal $\gamma^{\prime}$ distributions found in disk material might also prevent Orowan looping. As shown in this work, only in the case of a coarse uni-modal $\gamma^{\prime}$ distribution strained at $750^{\circ} \mathrm{C}$, load transfer from $\gamma$ to $\gamma^{\prime}$ was observed immediately at the onset of plastic yielding. In other cases, this load transfer was only observed after some plastic deformation, a regime difficult to study by transmission electron microscopy.

The in-situ loading experiment using neutron diffraction also allows one to plot the evolution of lattice misfit during plastic
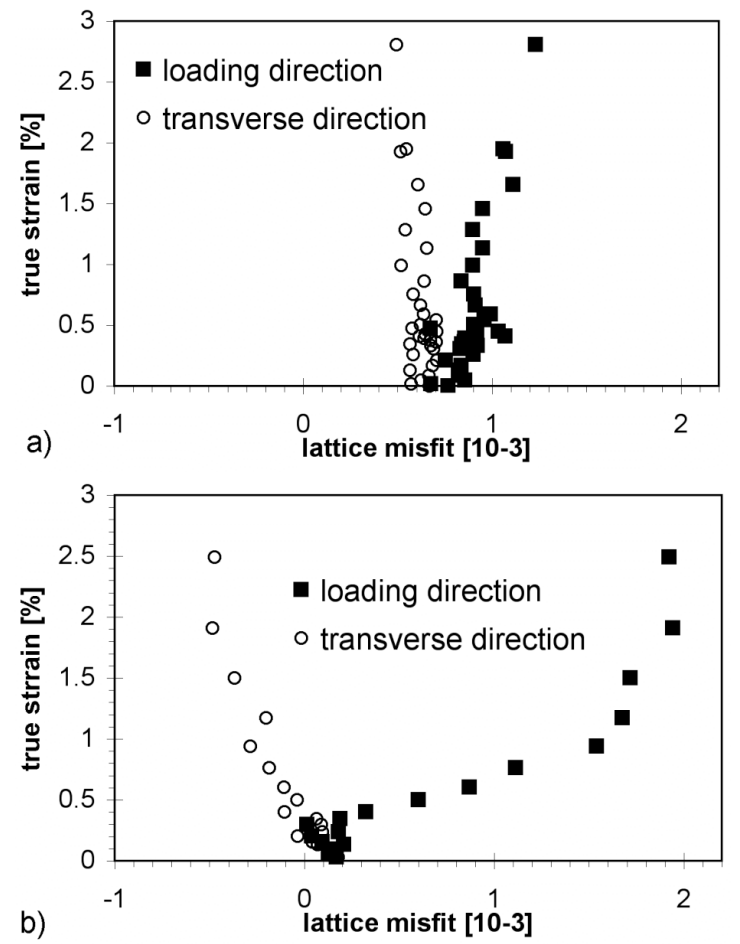

Figure 7: Lattice misfit in the loading and transverse direction as a function of true strain for the coarse $\gamma^{\prime}$ microstructure at (a) room temperature and (b) $750^{\circ} \mathrm{C}$. Note that the lattice misfit was measured in the loaded condition 
deformation. Since lattice misfit is directly related to any load transfer between the two phases, the lattice misfit becomes anisotropic if load transfer is observed. An example is given in Figure 7a and b, comparing the coarse $\gamma^{\prime}$ microstructure tested at room temperature (no load transfer) and at $750^{\circ} \mathrm{C}$ (strong load transfer). Here the lattice misfit is now plotted against the true strain recorded during the tensile experiments. Please note that in this case the lattice misfit was measured while the sample was loaded. In the case of the material tested at room temperature (Figure 7a) very little variation is seen between the lattice misfit measured in the loading and in the transverse direction. The slight difference observed between the two directions is simply a result of the uniaxial stress state. When tested at $750^{\circ} \mathrm{C}$, the early load transfer observed in Figure $6 \mathrm{c}$ results in a very substantial change of the lattice misfit for the loading and transverse direction even at less than $1 \%$ true strain. At the same time the lattice misfit becomes highly anisotropic with a maximum difference of almost $2.510^{-3}$ after $2.5 \%$ strain. It should be remembered that the change of lattice misfit is only a result of the observed load transfer, i.e. the substantial increase in lattice misfit is a product of load transfer and not the mechanistic reason for it. However, in terms of $\gamma^{\prime}$ stability during service, it can be seen that even small plasticity at high temperature for certain microstructures could result in highly anisotropic lattice misfit affecting long term $\gamma^{\prime}$ stability.

\section{Post mortem FEGSEM studies}

Only limited post mortem analysis has been carried out on the tested materials until now but further work is currently in progress. Before undertaking very time-consuming transmission electron microscopy studies, high resolution scanning electron microscopy was carried out on the tested samples. As mentioned above, the tested samples were cut in the middle of the gauge volume along the loading direction.

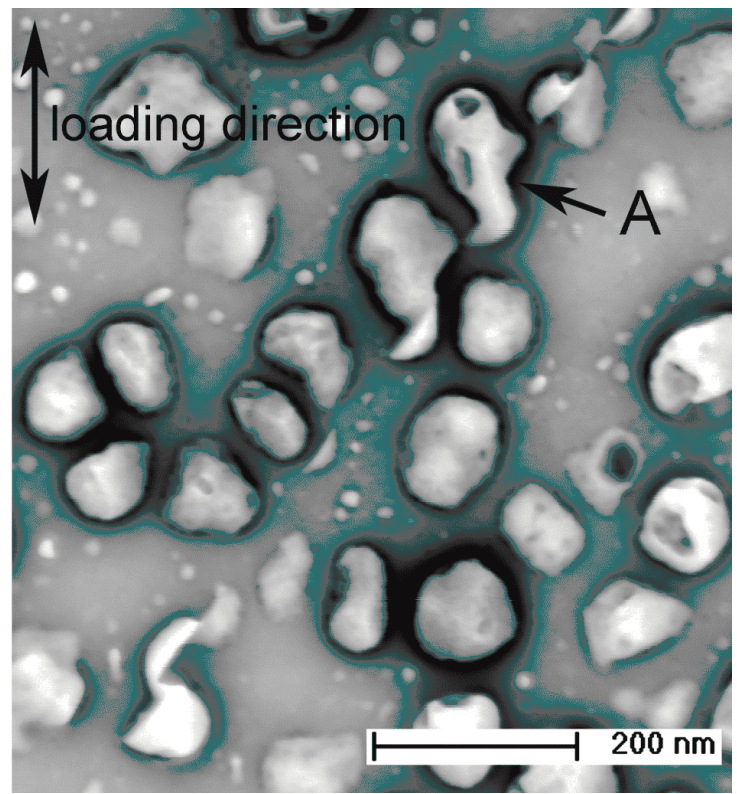

Figure 8: Post Mortem FEGSEM image of the fine $\gamma^{\prime}$ microstructure tested at $500^{\circ} \mathrm{C}$. The loading direction is indicated in the image. Sheared $\gamma^{\prime}$ particles can be clearly identified with a cutting direction close to $45^{\circ}$ to the loading direction (A).
Figure 8 is a high magnification image of the fine $\gamma^{\prime}$ microstructure tested at $500^{\circ} \mathrm{C}$ and strained to about $3 \%$ (see Figure $3 b$ ). The image seems to show a shear band cutting through several $\gamma^{\prime}$ particles. In this particular sample nearly every $\gamma$ grain contained these features. In most cases these bands were observed close to $45^{\circ}$ in respect of the loading axis. In comparison, the coarse $\gamma^{\prime}$ microstructures tested at the same temperature to approximately $5 \%$ strain and at $750^{\circ} \mathrm{C}$ to approximately $3 \%$ strain showed hardly any of these features. If these are indeed particles cut by dislocations, these observations support the assumption that the observed $\gamma^{\prime}$ load transfer in the coarse microstructure indicates Orowan looping rather than cutting by strongly coupled dislocations.

Further work is currently under way using a combination of electron backscatter diffraction (EBSD), Dual Beam Focused Ion Beam and TEM to identify possible Orowan loops in the coarse $\gamma^{\prime}$ microstructure tested at $750^{\circ} \mathrm{C}$

\section{Conclusions}

In situ loading experiments using neutron diffraction to measure load partitioning between $\gamma$ and $\gamma^{\prime}$ in RR1000 have been carried out at room temperature, $500^{\circ} \mathrm{C}$ and $750^{\circ}$. Instead of studying the material in a conventional condition, i.e. having a complex trimodal $\gamma^{\prime}$ size distribution, model microstructures with different uni-modal $\gamma^{\prime}$ size distributions have been generated. These unimodal $\gamma$ distributions were achieved by heat-treating the material above the $\gamma^{\prime}$-solvus followed by oil quenching and ageing at either $800^{\circ} \mathrm{C}, 900^{\circ} \mathrm{C}$ or $1050^{\circ} \mathrm{C}$. In the case of the fine $\gamma^{\prime}$ microstructure aged at $800^{\circ} \mathrm{C}$ it seems that a too fast cooling rate $\left(1^{\circ} \mathrm{C} / \mathrm{min}\right)$ from the aging temperature generated a bi-modal distribution. However, the two distributions were both significantly smaller than the medium and coarse $\gamma^{\prime}$ distributions. In this way it was then possible to study the effect of $\gamma^{\prime}$ particle size on the active deformation mechanisms between room temperature and $750^{\circ} \mathrm{C}$.

Neutron diffraction in-situ tensioning experiments were carried out to reveal the load sharing between $\gamma$ and $\gamma^{\prime}$. Further analysis of the intergranular strains will be used at a later stage to develop two different types of crystal plasticity models. The results shown here demonstrate that in-situ loading experiments using highly penetrating neutron radiation are a powerful tool for studying deformation mechanisms in nickel-base superalloys but that at the same time post mortem analysis of the deformed material using electron microscopy is vital to interpret certain aspects of the load sharing information.

The findings so far can be summarized as follows:

- The three different uni-modal model microstructures produced in RR1000 showed almost identical lattice misfit for a given temperature between $20^{\circ} \mathrm{C}$ and $750^{\circ} \mathrm{C}$ even though the aging temperatures varied by $250^{\circ} \mathrm{C}$. This can probably be attributed to the very slow cooling rates applied when the material was cooled from the aging temperature, generating a similar matrix and $\gamma^{\prime}$ chemistry for each microstructure. In general, the observed lattice misfit was about $1 / 3$ of what had been previously observed for Udimet $720 \mathrm{Li}$.

- When tensile loading the fine $\gamma^{\prime}$ microstructures between room temperature and $750^{\circ} \mathrm{C}$ no $\gamma$ to $\gamma^{\prime}$ load transfer was observed within the tested deformation range. This suggests 
that the $\gamma^{\prime}$ particles are being sheared by dislocations, which was confirmed by post mortem SEM studies

- When considering the first $2-3 \%$ of plastic deformation it was found that, at room temperature, no $\gamma$ to $\gamma^{\prime}$ load transfer is observed for any $\gamma^{\prime}$ particle size. As the testing temperature increases, load transfer can be observed first only for the coarse $\gamma^{\prime}$ microstructure and at $750^{\circ} \mathrm{C}$ for the medium and coarse $\gamma^{\prime}$ microstructure. This demonstrates that there is a combined particle size/temperature dependency for $\gamma$ to $\gamma^{\prime}$ load transfer.

- It was also observed in some cases that small levels of plastic deformation in the range of $1-3 \%$ did not result in any load transfer but upon further straining $\gamma$ to $\gamma^{\prime}$ load transfer started to occur. This suggests that in these cases $\gamma^{\prime}$ has a different hardening rate than the $\gamma$ matrix and that possibly cross slip onto the $\{100\}$ plane, which is associated with dislocation locking in the $\gamma^{\prime}$ phase, takes place at this stage.

- There are a number of mechanisms that could explain the observed $\gamma$ to $\gamma^{\prime}$ load transfer. Firstly, dislocation will get locked in $\gamma^{\prime}$ particles because of cross slipping onto the $\{100\}$ at increased test temperatures. This in turn could activate Orowan looping since it does not require dislocations to cut through $\gamma^{\prime}$. On the other hand, Orowan loops are rarely reported in polycrystalline nickel-base superalloys and it is generally believed that cutting of strongly coupled dislocations is the predominant deformation mechanism in coarse (below 400nm) $\gamma^{\prime}$ microstructures. At the moment it is not clear if this mechanism would also result in load transfer although this is likely since strong dislocation bowing is associated with this mechanism

- In some cases (mainly during room temperature testing) a slight $\gamma^{\prime}$ to $\gamma$ load transfer was observed, which has been previously reported for Udimet $720 \mathrm{Li}$ suggesting that the CRSS of $\gamma^{\prime}$ is lower than the CRSS of $\gamma$ at room temperature.

- As a result of the $\gamma$ to $\gamma^{\prime}$ load transfer, increasingly observed at high temperature and coarse $\gamma^{\prime}$ particles, the lattice misfit in the loading direction increases dramatically while in the transverse direction it turns negative. Such highly anisotropic lattice misfits were observed in the case of the coarse $\gamma^{\prime}$ microstructure tested at $750^{\circ} \mathrm{C}$ after only $1 \%$ strain (which includes the elastic part of the loading).

\section{Acknowledgement}

The authors would like to thank the EPSRC (EP/E020933/1), MoD (joint grant scheme) and Rolls-Royce plc. for financially supporting this project and the ISIS facility, UK for providing the necessary neutron diffraction beam time and in-situ loading devices. The authors are also particularly grateful for advice and fruitful discussions with Rob Mitchell (Rolls-Royce plc.), Mike Winstone (DSTL) and Professor Ben Mori (University of Manchester)

\section{References}

[1]: Sims, CT, Stolokoff NS, and Hagel, WC, Superalloys II, 1987, John Wiley \& Sons.
[2]: R.J. Mitchell, M. Preuss, and M.J. Hardy, "Inter-relationships between composition, $\gamma^{\prime}$, morphology, hardness, and $\gamma-\gamma^{\prime}$ mismatch in advanced polycrystalline nickel-base superalloys during aging at $800^{\circ} \mathrm{C}$ ", Metallurgical and Materials Transactions A, 38A (2007), 615-627

[3]: R.J. Mitchell, M. Preuss, M.J. Hardy, S. Tin, "Influence of Composition and Cooling Rate on Constrained and Unconstrained Lattice Parameters in Advanced Polycrystalline Nickel-Base Superalloys", Materials Science and Engineering A, 423 (2006), 282-291

[4]: R.J. Mitchell, M. Preuss, S. Tin and M.C. Hardy, "The influence of cooling rate from temperatures above the $\gamma^{\prime}$ solvus on morphology, mismatch and hardness in advanced polycrystalline nickel-base superalloys", Materials Science and Engineering A, 473 (2008), 158-165

[5]: B. Reppich, "Some new aspects concerning particle hardening mechanisms in $\gamma^{\prime}$ precipitating Ni-base alloys-I. Theoretical concept", Acta Metallurgica, 30 (1982), 87-94

[6]: B. Reppich, P. Schepp, G. Wehner, "Some new aspects concerning particle hardening mechanisms in $\gamma^{\prime}$ precipitating nickel-base alloys-II. Experiments", Acta Metallurgica, 30 (1982), 95-104

[7]: G.N. Maniar, Metallurgical Transaction, 1970; 1: 31-42.

[8]: J.H. Westbrook, "Temperature dependence of the hardness of secondary phases common in turbine bucket alloys", Transaction of the American Institute of Mining and Metallurgical Engineers, 209 (1957), 898-904

[9]: P.H. Thornton, R.G. Davies, and T.L. Johnston, "Temperature dependence of flow stress of $\gamma$ and phase based upon $\mathrm{Ni}_{3} \mathrm{Al}^{\prime}$ ", Metallurgical Transaction, 1 (1970), 207-218.

[10]: A.E. Staton-Bevan, R.D. Rawlings, "Deformation behaviour of single-crystal $\mathrm{Ni}_{3}(\mathrm{Al}, \mathrm{Ti})$ ", Physica Status Solidi A Applied Research, 29 (1975), 613-622.

[11]: J.S. Kohler, F. Seitz, J. Appl. Mech., 1947; 14: A217-A224.

[12]: R.J. Taunt, B. Ralph, "Observations of fine-structure of superdislocations in $\mathrm{Ni}_{3} \mathrm{Al}$ by field ion microscopy, Philosophical Magazine, 30 (1974), 1379-1394.

[13]: N. Clement, A. Couret, D. and Caillard, Philosophical Magazine A, 64 (1991), 669-694.

[14]: M.R. Daymond, M. Preuss and B. Clausen: Evidence of variation in slip mode in a polycrystalline nickel-base superalloy with change in temperature from neutron diffraction strain measurements, Acta Materialia, 55 (2007), 3089-3102

[15]:W.M. Kane, U. Krupp, T. Jacobs and C.J. McMahon: On the mechanism of quench cracking in Rene 95 nickel-based superalloy, Materials Science and Engineering: A, 402 (2005), $42-46$

[16]: M. Preuss, J.W.L. Pang, P.J. Withers, G.J. Baxter: Inertia Welding Nickel-based Superalloy. Part I: Metallurgical Characterization, Metallurgical and Materials Transactions A, 33A (2002), 3115-3225

[17]: Noyan, I.C. and Cohen, J.B., Residual Stress - Measurement by Diffraction and Interpretation. Materials Research and Engineering, (New York: Springer-Verlag, 1987), 272.

[18]: Fitzpatrick, M.E. and Lodini, A., eds. Analysis of Residual Stress by Diffraction using Neutron and Synchrotron Diffraction. 2003, Taylor and Francis: London. 348.

[19]: J.R. Santisteban, M.R. Daymond, J.A. James and L Edwards, "ENGIN-X: a third generation neutron strain scanner", Journal of Applied Crystallography, 39 (2006), 812-825

[20]: M.R. Daymond and H.G. Priesmeyer, “ Elastoplastic deformation of ferritic steel and cementite studied by neutron 
diffraction and self-consistent modeling", Acta Materialia, 50 (2002), 1613-1626.

[21]: M.R. Daymond and P.J. Withers, "A synchrotron radiation study of transient internal strain changes during the early stages of thermal cycling in an $\mathrm{Al} / \mathrm{SiC}_{\mathrm{w}} \mathrm{MMC}$ "Scripta Materialia, 35 (1996), 717-720.

[22]: Windsor, C.G., Pulsed neutron scattering, (Taylor and Francis, London, 1981).

[23]: H.M Rietveld, "A profile refinement method for nuclear and magnetic structures", Journal of Applied Crystallography, 2 (1969), 65-71.

[24]: L. B. McCusker, R. B. Von Dreele, D. E. Cox, D. Louër and P. Scardi, "Rietfeld refinement guidelines", Journal of Applied Crystallography, 32 (1999), 36-50

[25]: GSAS Manual, A.C.Larson and R.B.Von Dreele

[26]: V.F.Sears and S.A.Shelley, "Debye-Waller factor for elemental crystals", Acta Crystallographica Section, A47 (1991), 441-446

[27]: R.F.Miller, G.S. Ansell, "Low temperature mechanical behavior of Ni-15Cr-Al-Ti-Mo alloys", Metallurgical Transaction A - Physical Metallurgy and Materials Science, 8(1977), 19791991.

[28]: M. Heilmaier, U. Leetz and B. Reppich, "Order strengthening in the cast nickel-based superalloy IN 100 at room temperature", Materials Science and Engineering A, 319-321 (2001), 375-378

[29]: J.E. King, "Fatigue crack propagation in nickel-base superalloys - effect of microstructure, load ratio and temperature", Materials Science and Technology, 3 (1987), 750764. 